\title{
A nonlinear relationship between low-density-lipoprotein cholesterol levels and atrial fibrillation among patients with hypertension in China
}

\author{
Jia-Yi Huang ${ }^{1}$, Lin Liu ${ }^{2}$, Yu-Ling Yu ${ }^{2}$, Chao-Lei Chen ${ }^{2}$, Kenneth Lo ${ }^{2,3}$, Bin Zhang ${ }^{2}$, Song-Tao Tang ${ }^{4}$, \\ Yu-Qing Huang', Ying-Qing Feng ${ }^{1}$
}

${ }^{1}$ Guangdong Provincial People's Hospital, School of Medicine, South China University of Technology, Guangzhou, China; ${ }^{2}$ Department of Cardiology, Guangdong Provincial Key Laboratory of Coronary Heart Disease Prevention, Guangdong Cardiovascular Institute, Guangdong Provincial People's Hospital, Guangdong Academy of Medical Sciences, School of Medicine, South China University of Technology, Guangzhou, China; ${ }^{3}$ Centre for Global Cardiometabolic Health, Department of Epidemiology, Brown University, Providence, RI, USA; ${ }^{4}$ Community Health Center of Liaobu County, Dongguan, China

Contributions: (I) Conception and design: All authors; (II) Administrative support: YQ Huang, YQ Feng; (III) Provision of study materials or patients: All authors; (IV) Collection and assembly of data: All authors; (V) Data analysis and interpretation: L Liu, YQ Huang; (VI) Manuscript writing: All authors; (VII) Final approval of manuscript: All authors.

Correspondence to: Prof. Ying-Qing Feng. Guangdong Provincial People's Hospital, School of Medicine, South China University of Technology, No. 106, Zhongshan Second Road, Yuexiu District, Guangzhou 510080, China. Email: 651792209@qq.com.

Background: The association between low-density-lipoprotein cholesterol (LDL-C) and atrial fibrillation (AF) in hypertensive population remains controversial. Therefore, we explored the relationship between LDL-C and AF among patients with hypertension in a Chinese community.

Methods: This is a retrospective cross-sectional study that enrolled 7,808 hypertensive patients between January 2013 and December 2013 in Guangdong, China. AF was diagnosed by 12-lead electrocardiogram (ECG) or self-reported status. LDL-C value were categorized by quartiles. Univariate and multivariate logistic regression were performed to examine the relation between LDL-C and AF. LDL-C values were expressed in continuous (every $1 \mathrm{mg} / \mathrm{dL}$ increment) or categorical variables in each regression model.

Results: Among 7,808 (47.1\% man, with mean age 62.3 years) participants, $78 \mathrm{AF}$ cases were identified. In multivariate logistic regression, when LDL-C was presented as continuous variable, it was inversely associated with the occurrence of new onset $\mathrm{AF}(\mathrm{OR}=0.99$, 95\% CI: 0.98, 1.00; $\mathrm{P}=0.018)$. Meanwhile, when LDL-C was presented as categorical variable, the negative association between LDL-C and AF was attenuated after adjusting for confounders. Adjusted restricted cubic spline demonstrated a non-linear correlation between LDL-C and AF.

Conclusions: Lower levels of LDL-C was associated with increased incidence of AF in a Chinese community hypertensive population.

Keywords: Atrial fibrillation (AF); hypertension; low-density-lipoprotein cholesterol

Submitted Feb 19, 2020. Accepted for publication Jul 14, 2020.

doi: 10.21037/apm-20-451

View this article at: http://dx.doi.org/10.21037/apm-20-451

\section{Introduction}

Hypertension is one of the most common chronic diseases in worldwide (1). In China, 23.2\% (approximately 244.5 million) of the adult population has hypertension (2).
Elevated blood pressure can lead to numerous target organs damage and related complications such as heart failure, stroke, chronic kidney disease, and death (3). Meanwhile, atrial fibrillation (AF) as a common complication of 
hypertension (4), which can cause cardiovascular events such as stroke $(5,6)$. The estimated number of individuals with AF in the world was 33.5 million in 2010 (7). In a large cohort prospective study that included 34,221 initially healthy women. After 12.4 years of follow up, 644 incident of AF events occurred and demonstrated blood pressure (BP) was strongly associated with a higher risk of AF (8). Similar result was found in another study with 35 years of followup that included 2014 initially healthy men, systolic BP $\geq 140 \mathrm{mmHg}$ had 1.6-fold (95\% CI: 1.15-2.21) risk of AF compared with those with systolic $\mathrm{BP}<128 \mathrm{mmHg}$ (9).

However, the mechanism of $\mathrm{AF}$ remains unclear now. A large number of previous studies have shown that $\mathrm{AF}$ was a multifactorial disease, and demonstrated that gender, race, obesity, and high density lipoprotein cholesterol (HDL-C) were all closely related to AF $(4,10-12)$. Previous studies also manifested AF was closely associated with inflammatory response and oxidative stress (13-15). Lowdensity-lipoprotein cholesterol (LDL-C) was also reported to be related to inflammatory and oxidative stress $(16,17)$. Meanwhile, several studies have identified that LDL-C had negative association with $\mathrm{AF}(18,19)$, and some identified null association. (20).

In brief, the epidemiological evidence on the association between LDL-C and the risk of AF is still inconsistent (18-21), especially among people with hypertension. Therefore, we investigated the relationship between LDL-C and AF in a group of hypertensive patients in China. We present the following article in accordance with the STROBE reporting checklist (available at http:// dx.doi.org/10.21037/apm-20-451).

\section{Methods}

\section{Study population and design}

This was a cross-sectional study in one Chinese community. The population cohort comprised of 8,169 hypertensive patients aged $\geq 18$ years old, who were attending annual physical examinations in the community health care center in Liaobu, Guangdong, China. Participants were consecutively enrolled during January 2013 and December 2013. All enrolled participants were diagnosed with essential hypertension and had complete sets of data. The participants who were did not provide data of BP $(n=38)$, blood lipid $(\mathrm{n}=245)$, serum creatinine $(\mathrm{n}=255)$, demographic features $(n=45)$, or data of electrocardiograph (ECG) $(n=71)$ were excluded (Figure 1). Finally, 7,808 subjects were included

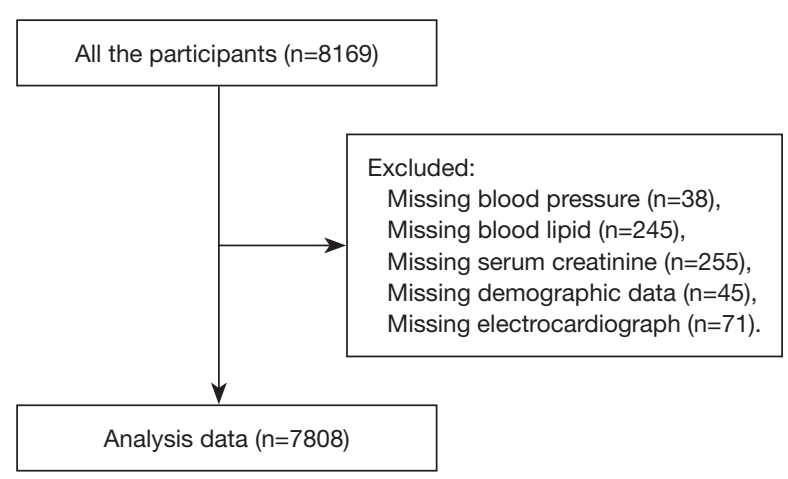

Figure 1 The flowchart of selecting participants for analysis.

in the data analysis. The study was in compliance with the principles outlined in the Declaration of Helsinki (as revised in 2013) and was approved by the institutional medical ethical committee (NO. GDREC2012143H). Written informed consent was obtained from all participants.

\section{Measurement of covariates}

At baseline, a structured questionnaire was administered by trained study personnel to acquire demographic characteristics, including demographic data, medical history [beta-blockers, calcium channel blockers (CCB), angiotensin-converting enzyme inhibitors (ACEI)/ angiotensin-receptor blockers (ARB), statins), and medical history (coronary heart disease (CAD), diabetes mellitus (DM), stroke]. Parameters for physical measurements included systolic BP (SBP), diastolic BP (DBP), and body mass index (BMI). Blood samples were collected after at least 8 hours of fasting. Fasting blood glucose (FBG), total cholesterol (TC), triglyceride (TG), LDL-C, HDL-C was measured using standardized laboratory methods. Hypertension was defined as $\mathrm{SBP} \geq 140 \mathrm{mmHg}$, and/ or DBP $\geq 90 \mathrm{mmHg}$, and/or the use of antihypertensive medicine within 2 weeks, with reference to 2010 Chinese guidelines for the management of hypertension (2). ECG was standard resting 12-lead recordings at a paper speed of $50 \mathrm{~mm} / \mathrm{s}$ and a calibration of $1 \mathrm{mV}$ per $10 \mathrm{~mm}$ and were collected from all subjects at the baseline. Participants were considered to have AF if AF rhythm have been captured in ECG. AF rhythm was defined as (I) irregular R-R intervals, (II) absence of distinct repeating $\mathrm{P}$ waves, (III) irregular atrial activity show on ECG, according to 2014 ACC/AHA/ HRS Guideline for the Management of Patients With Atrial Fibrillation (4). Left ventricular hypertrophy (LVH) 
was defined as Cornell criteria ( $\mathrm{S}$-wave in V3 and $\mathrm{R}$-wave in aVL > $24 \mathrm{~mm}$ in men, and $\mathrm{S}$ in $\mathrm{V} 3$ and $\mathrm{R}$ in aVL > $20 \mathrm{~mm}$ in women) and the Sokolow-Lyon criteria (tallest $\mathrm{R}$ wave in V5 or V6 and S-wave in V1>35 mm) with or without concomitant strain pattern ( $\mathrm{T}$-wave inversion in lateral leads) (22). All ECG results were identified and diagnosed by qualified practitioners. Estimated glomerular filtration rate (eGFR) was calculated from the simplified MDRD equation (23).

\section{Statistical analysis}

Continuous variables were expressed as means \pm standard deviations, while categorical variables were presented as absolute values and percentages. One-way ANOVA, Kruskal-Wallis $H$ test and chi-square test were performed to determine any significant subgroup differences According to LDL-C levels in quartile (Q1, Q2, Q3, Q4). Univariate and multivariate logistic regression were performed to evaluate the association between LDL-C and AF, and the results were reported as odds ratio (OR) with $95 \%$ confidence intervals (CI). LDL-C values were treated as both continuous (every $1 \mathrm{mg} / \mathrm{dL}$ increment) or categorical variables in different regression models. Model 1 only included LDL-C values. Model 2 was adjusted for age, sex and SBP. Meanwhile, Model 3 was adjusted for age, eGFR, sex, diabetes, CAD, SBP, smoking, drinking, FBG, TC, TG, LDL-C, HDL-C, antihypertensive drugs and statin. To visualize the relationship between LDL-C and AF, we fitted restricted cubic spine by adjusting for potential confounders. A two-tailed P value less than 0.05 was considered as significant. Data management and analyses were performed using the statistical software package $\mathrm{R}$ version 3.32 (http://www.R-project.org, The R Foundation, Vienna, Austria).

\section{Results}

\section{Baseline characteristics}

Baseline characteristics for participants were summarized in Table 1. The present analysis included 7,808 participants, including 3,678 men (47.1\%). The average age was $62.3 \pm 13.7$ years. A total of 78 AF cases were identified, including 42 men (53.8\%) and average age were $72.3 \pm 11.3$ years. Participants with AF were older, had higher prevalence of CAD and had lower levels of LDL-C, TG and TC compared to those without AF (all $\mathrm{P}<0.05$ ).
The statin usage was significantly higher in AF group than in non-AF group.

Table 2 showed baseline characteristics according to LDL-C quartiles (Q1: LDL-C <78.9 mg/dL, Q2: $78.9-$ $96.6 \mathrm{mg} / \mathrm{dL}, \mathrm{Q} 3:$ 96.6-115.6 mg/dL, Q4: $\geq 115.6 \mathrm{mg} / \mathrm{dL})$. Each group had 1,952 subjects. We found that age, sex, SBP, DBP, BMI, eGFR, TC, TG, HDL-C, diabetes, CAD, stroke and statin consumption were significantly different among four LDL-C groups (all $\mathrm{P}<0.05$ ). However, there was no statistically significant difference in smoking, drinking, FBG, LVH among four groups (all $\mathrm{P}>0.05$ ).

\section{The association between $L D L-C$ and $A F$}

As shown in Table 3, LDL-C (OR =0.99, 95\% CI: 0.98, 0.99, $\mathrm{P}=0.001)$, SBP $(\mathrm{OR}=0.96,95 \% \mathrm{CI}: 0.94,0.98, \mathrm{P}=0.001)$, TC $(\mathrm{OR}=0.99,95 \% \mathrm{CI}: 0.98,1.0, \mathrm{P}=0.001), \mathrm{TG}(\mathrm{OR}=1.0$, 95\% CI: 0.99,1.00, $\mathrm{P}=0.009)$ were negatively associated with AF. Meanwhile, without taking statin $(\mathrm{OR}=2.06$, 95\% CI: $1.24,3.40, \mathrm{P}<0.01)$ was positively associated with the incident of AF. In addition, the multivariate logistic regression demonstrated LDL-C was still significantly inversely with $\mathrm{AF}(\mathrm{OR}=0.98,95 \% \mathrm{CI}$ : 0.97, 1.00; $\mathrm{P}=0.045)$.

\section{The non-linear relationship between $L D L-C$ and $A F$}

As shown in Table 4, when LDL-C was presented as a continuous variable, it was inversely associated with $\mathrm{AF}$ in all regression models (Model 1: OR: 0.99, 95\% CI: 0.98-0.99; Model 2: OR: 0.99, 95\% CI: 0.98-0.99; Model 3: OR: 0.99, 95\% CI: 0.98-1.00; all $\mathrm{P}<0.05)$. When LDL-C was presented as categorical variable, when using Q1 as reference, the OR was 0.51 (95\% CI: 0.28-0.92) for Q2, 0.33 (95\% CI: $0.17-0.65$ ) for Q3, and 0.51 for Q3 (95\% CI: 0.28-0.92) $(\mathrm{P}<0.05)$ in model 1. However, after fully adjusted for potential confounding factors, Q2 and Q4 had no significant statistical association with AF $(\mathrm{P}>0.05)$, but Q3 was negatively related with AF (OR: 0.40, 95\% CI: $0.20-0.81, \mathrm{P}=0.011$ ) in model 3 . To better demonstrate the relation between $\mathrm{LDL}-\mathrm{C}$ and $\mathrm{AF}$, we fitted the restricted cubic spline for LDL-C, and a non-linear relationship was found after adjusting for multiple confounders (Figure 2).

\section{Discussion}

In the present study, we found that LDL-C was inversely associated with AF. Interestingly, when LDL-C was 
Table 1 Baseline characteristics of participant patients

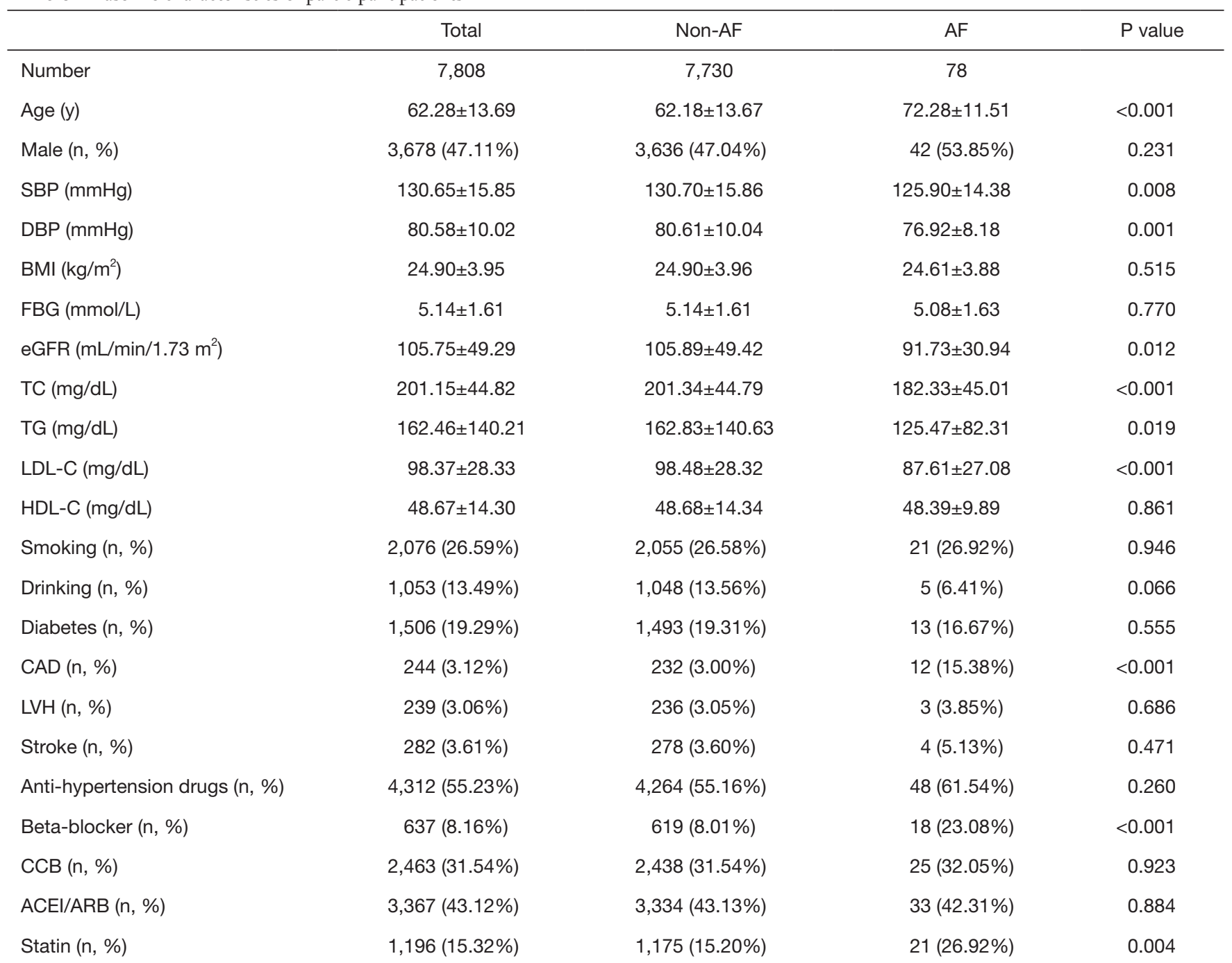

Data are expressed as mean \pm standard deviation or percentage. AF, atrial fibrillation; CAD, coronary heart disease; LVH, left ventricular hypertrophy; CCB, calcium channel blockers; ACEI/ARB, angiotensin-converting enzyme inhibitors/angiotensin-receptor blockers; SBP, systolic blood pressure; DBP, diastolic blood pressure; BMI, body mass index; FBG, fasting blood glucose; TG, triglyceride; TC, total cholesterol; LDL-C, low density lipoprotein cholesterol; HDL-C, high density lipoprotein cholesterol; eGFR, estimated glomerular filtration.

presented as categorical variables, the negative association between LDL-C and AF was attenuated.

It has been demonstrated in a secondary analysis of the Antihypertensive and Lipid-Lowering Treatment to Prevent Heart Attack Trial (ALLHAT) which included 14,837 participants. They found that LDL-C was lower in the participants with $\mathrm{AF} /$ atrial flutter (AFL) group than the group without $\mathrm{AF} / \mathrm{AFL}(\mathrm{P}<0.001)(24)$. Our results also indicted that $\mathrm{AF}$ patients have lower LDL-C compared to patients without AF. Although AFL was not measured in the present study, the results are still consistent because the prevalence of AFL is much lower than that of $\mathrm{AF}$ (25).

In our analysis, the inverse association between LDL-C and $\mathrm{AF}$ was independent of multiple risk factors. Similar result was found in the Atherosclerosis Risk in Communities Study (ARIC) (26). When compared to LDL-C level $<2.59 \mathrm{mmol} / \mathrm{L}(100.15 \mathrm{mg} / \mathrm{dL})$, people with LDL-C level ranged $2.59-4.13 \mathrm{mmol} / \mathrm{L}(100.15-159.71 \mathrm{mg} / \mathrm{dL})$ had lower risk of $\mathrm{AF}$ (HR $=0.84,95 \% \mathrm{CI}: 0.72-0.97)$. Also, a prospective cohort study in Kailuan, China, suggested that LDL-C $>110 \mathrm{mg} / \mathrm{dL}$ was inversely associated with $\mathrm{AF}$ (HR $=0.60,95 \%$ CI: 0.43-0.83). However, participants 
Table 2 Baseline characteristics of participant patients by LDL-C quartiles

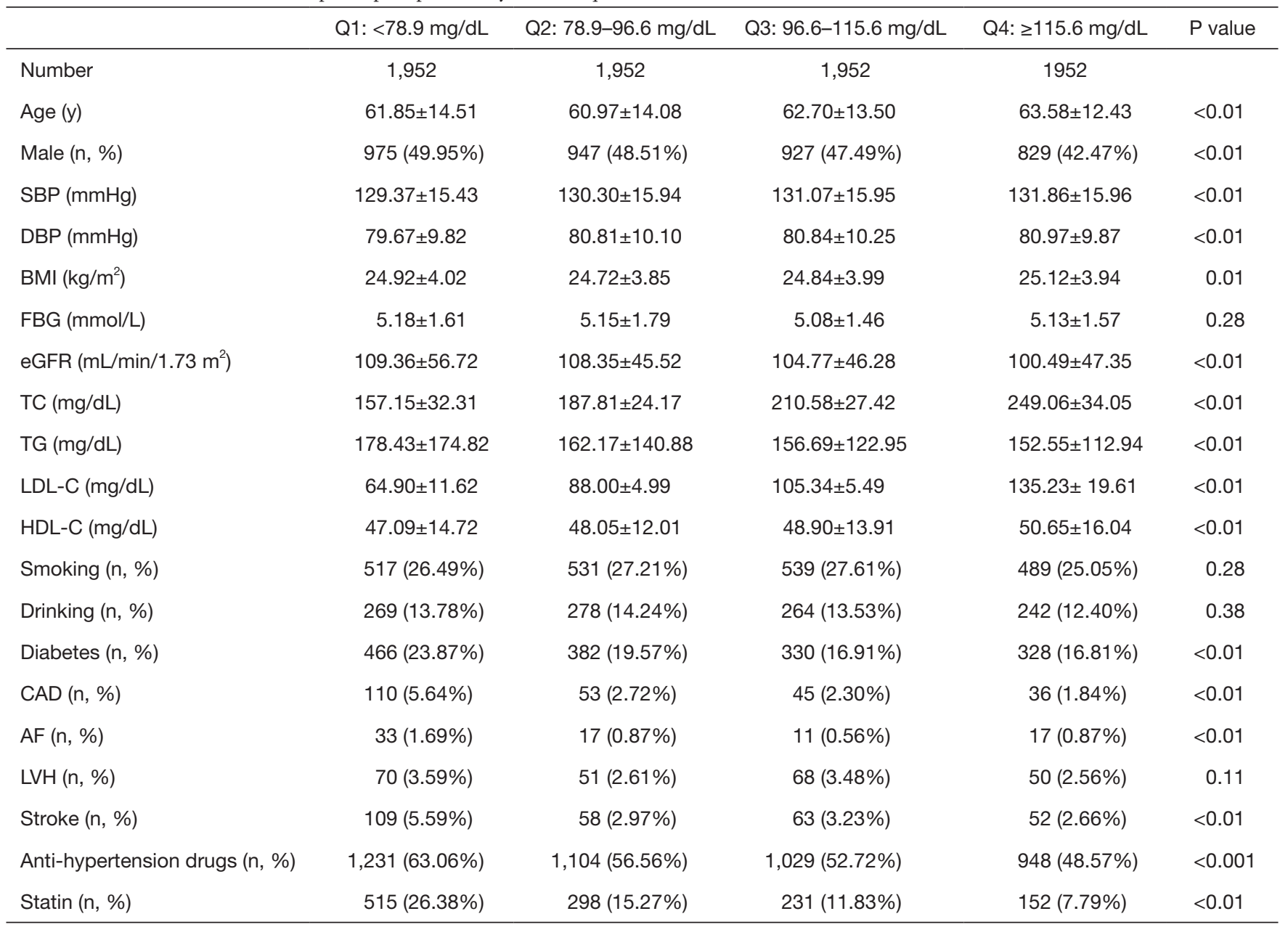

Data are expressed as mean \pm standard deviation or percentage. SBP, systolic blood pressure; DBP, diastolic blood pressure; BMI, body mass index; FBG, fasting blood glucose; eGFR, estimated glomerular filtration; TC, total cholesterol; TG, triglyceride; LDL-C, low density lipoprotein cholesterol; HDL-C, high density lipoprotein cholesterol; CAD, coronary heart disease; AF, atrial fibrillation; LVH, left ventricular hypertrophy; CCB, calcium channel blockers; ACEl/ARB, angiotensin-converting enzyme inhibitors/angiotensin-receptor blockers.

LDL-C $\leq 110 \mathrm{mg} / \mathrm{dL}$ did not have significantly higher risk for $\operatorname{AF}(19)$.

Moreover, an analysis on the Multi-Ethnic Study of Atherosclerosis (MESA) and the Framingham Heart Study (FHS) showed that LDL-C was not associated with the risk of AF (MESA: HR $=1.15$, 95\% CI: $0.99-1.35$; FHS: HR $=0.95,95 \%$ CI: $0.82-1.09$; combined: HR $=1.04,95 \%$ CI: $0.94-1.15)$ in the fully adjusted model (20). The regional and ethnical difference may account for the inconsistent findings. In addition, confounders being adjusted may not be the same across studies.

Although the exact mechanisms between LDL-C and AF remains unresolved, several mechanisms may help to explain the inverse association. First, some researchers considered that high levels of LDL-C might have an anti-inflammatory effect $(27,28)$, and AF was closely associated with inflammatory reaction (15). Low level of LDL-C probably contributes to the pathogenesis of AF via enhancing inflammatory response. Second, membrane lipid composition is the main determinant of cell excitability, previous vitro studies have identified that cholesterol regulates the function of ion channels, such as $\mathrm{Kv} 1.5 \mathrm{~K}+$ channel, $\mathrm{Na}+$ channel in myocardial cell. Lowered cholesterol levels reduce cell excitability which might be potentially contributing to the development of AF $(29,30)$. Third, subclinical hyperthyroidism decreases LDL-C (31) and is also associated with increased risk of $\mathrm{AF}$ (32). In addition, a latest article purposed the concept 
Table 3 Univariate and multivariate logistic regression for the relationship between clinical variables and AF

\begin{tabular}{|c|c|c|}
\hline & Univariate, OR $(95 \% \mathrm{Cl}), \mathrm{P}$ value & Multivariate, OR $(95 \% \mathrm{Cl}), \mathrm{P}$ value \\
\hline \multicolumn{3}{|l|}{ Gender } \\
\hline Male & 1.0 & 1.0 \\
\hline Female & $0.76(0.49,1.19), 0.232$ & $0.52(0.30,0.91), 0.022$ \\
\hline No & 1.0 & 1.0 \\
\hline Yes & $1.02(0.62,1.68), 0.946$ & $0.74(0.40,1.40), 0.359$ \\
\hline \multicolumn{3}{|l|}{ Drinking } \\
\hline No & 1.0 & 1.0 \\
\hline No & 1.0 & 1.0 \\
\hline Yes & $0.84(0.46,1.52), 0.556$ & 0.85 (0.45, 1.60), 0.616 \\
\hline \multicolumn{3}{|l|}{ LVH } \\
\hline No & 1.0 & 1.0 \\
\hline Yes & $1.27(0.40,4.06), 0.687$ & 0.97 (0.30, 3.17), 0.959 \\
\hline \multicolumn{3}{|l|}{ Statin } \\
\hline Yes & 1.0 & 1.0 \\
\hline No & $2.06(1.24,3.40), 0.005$ & $1.58(0.92,2.74), 0.100$ \\
\hline $\mathrm{TC}(\mathrm{mg} / \mathrm{dL})$ & $0.99(0.98,1.00), 0.001$ & $1.00(0.99,1.01), 0.505$ \\
\hline $\mathrm{TG}(\mathrm{mg} / \mathrm{dL})$ & $1.00(0.99,1.00), 0.009$ & $1.00(0.99,1.00), 0.102$ \\
\hline LDL-C (mg/dL) & 0.99 (0.98, 0.99), 0.001 & $0.98(0.97,1.00), 0.045$ \\
\hline $\mathrm{HDL}-\mathrm{C}(\mathrm{mg} / \mathrm{dL})$ & $1.00(0.98,1.02), 0.860$ & $1.00(0.98,1.02), 0.777$ \\
\hline
\end{tabular}

AF, atrial fibrillation; CAD, coronary heart disease; LVH, left ventricular hypertrophy; Beta, beta-blockers; CCB, calcium channel blockers; ACEI/ARB, angiotensin-converting enzyme inhibitors/angiotensin-receptor blockers; SBP, systolic blood pressure; DBP, diastolic blood pressure; BMI, body mass index; FBG, fasting blood glucose; eGFR, estimated glomerular filtration; TC, total cholesterol; TG, triglyceride; LDL-C, low density lipoprotein cholesterol; HDL-C, high density lipoprotein cholesterol.

of cardiac lipotoxicity in which heart tissue uptake increased lipoproteins, therefore the circulatory lipids may be reduced. The imbalance of lipids distribution among tissue and circulation may be another explanation for the paradoxical relation between LDL-C and AF (33).
Some limitations should be considered when interpreting our findings. First, this was a cross-sectional study, which indicate correlation but not causation. Second, in the present study, AF was diagnosed by ECG at the baseline but not by the 24 hours ECG, which might miss out 
Table 4 The results of two-piecewise linear regression model between LDL-C and AF

\begin{tabular}{lccc}
\hline & Model I, OR $(95 \% \mathrm{Cl}), \mathrm{P}$ value & Model II, OR $(95 \% \mathrm{Cl}), \mathrm{P}$ value & Model III, OR (95\% Cl), P value \\
\hline LDL-C (every 1 mg/dL increased) & $0.99(0.98,0.99), 0.001$ & $0.99(0.98,0.99), 0.001$ & $0.99(0.98,1.00), 0.018$ \\
LDL-C groups & & & 1.0 \\
Q1 & 1.0 & $0.56(0.31,1.02), 0.058$ & $0.64(0.35,1.17), 0.145$ \\
Q2 & $0.51(0.28,0.92), 0.025$ & $0.33(0.17,0.66), 0.002$ & $0.40(0.20,0.81), 0.011$ \\
Q3 & $0.33(0.17,0.65), 0.002$ & $0.52(0.29,0.95), 0.033$ & $0.66(0.35,1.25), 0.204$ \\
Q4 & $0.51(0.28,0.92), 0.025$ & 0.007 & 0.046 \\
P for trend & 0.007 & & \\
\hline
\end{tabular}

Adjusted for age, sex, smoking, drinking, diabetes, CAD, LVH, stroke, beta-blocker, CCB, ACEI/ARB, stains, SBP, DBP, BMI, FBG, eGFR, TC, TG and HDL-C. OR, odd ratio, Cl, confidence interval. AF, atrial fibrillation; CAD, coronary heart disease; LVH, left ventricular hypertrophy; Beta, beta-blockers; CCB, calcium channel blockers; ACEI/ARB, angiotensin-converting enzyme inhibitors/angiotensinreceptor blockers; SBP, systolic blood pressure; DBP, diastolic blood pressure; BMI, body mass index; FBG, fasting blood glucose; eGFR, estimated glomerular filtration; TC, total cholesterol; TG, triglyceride; LDL-C, low density lipoprotein cholesterol; HDL-C, high density lipoprotein cholesterol.

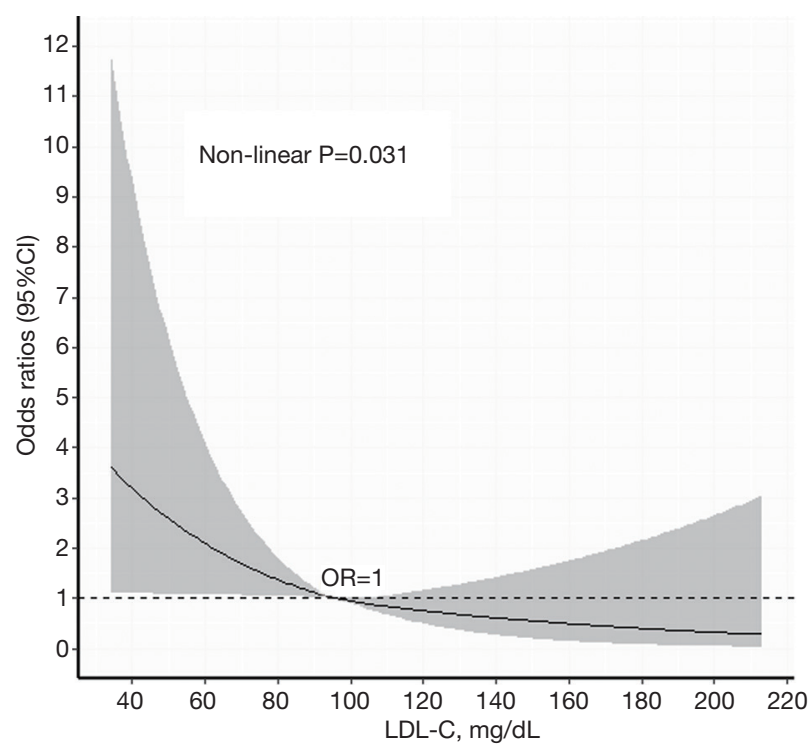

Figure 2 Restricted cubic spline of low-density lipid cholesterol levels and atrial fibrillation. Adjusted for age, sex, smoking, drinking, diabetes, CAD, LVH, stroke, Beta-blocker, CCB, ACEI/ ARB, statin, SBP, DBP, BMI, FBG, eGFR, TC, TG and HDL-C. The solid curve shows the association between LDL-C levels and AF. AF, atrial fibrillation; CAD, coronary heart disease; $\mathrm{LVH}$, left ventricular hypertrophy; Beta, beta-blockers; CCB, calcium channel blockers; ACEI/ARB, angiotensin-converting enzyme inhibitors/ angiotensin-receptor blockers; SBP, systolic blood pressure; DBP, diastolic blood pressure; BMI, body mass index; FBG, fasting blood glucose; eGFR, estimated glomerular filtration; TC, total cholesterol; TG, triglyceride; LDL-C, low density lipoprotein cholesterol; HDL-C, high density lipoprotein cholesterol. paroxysmal or subclinical AF and lead to the relatively low incidence of AF. Third, we did not examine the levels of inflammatory biomarkers, and did not performed tests on thyroid function, heart function, and did not obtain the data of central obesity. Forth, despite the adjustment of multiple confounders, effects of residual confounders may exist, such as the impact of uric acid and sleep. Finally, this was a single center study, therefore the conclusions could not be directly extrapolated to other populations.

In conclusion, we found that lower levels of LDL-C were associated with increased incidence of $\mathrm{AF}$ in a Chinese community hypertensive population. However, when LDL-C was presented as categorical variables, after adjusted potential confounders, the negative association between LDL-C and AF was attenuated.

\section{Acknowledgments}

Funding: This work was supported by the Science and Technology Program of Guangzhou (No. 201604020143, No. 201604020018 , No. 201604020186 , and No. 201803040012), and the National Key Research and Development Program of China (No. 2017YFC1307603, No. 2016YFC1301305), the Key Area R\&D Program of Guangdong Province (No. 2019B020227005).

\section{Footnote}

Reporting Checklist: The authors have completed the 
STROBE reporting checklist. Available at http://dx.doi. org/10.21037/apm-20-451

Data Sharing Statement: Available at http://dx.doi. org/10.21037/apm-20-451

Peer Review File: Available at http://dx.doi.org/10.21037/ apm-20-451

Conflicts of Interest: All authors have completed the ICMJE uniform disclosure form (available at http://dx.doi. org/10.21037/apm-20-451). The authors report grants from Science and Technology Program of Guangzhou, grants from National Key Research and Development Program of China, grants from Key Area R\&D Program of Guangdong Province, during the conduct of the study.

Ethical Statement: The authors are accountable for all aspects of the work in ensuring that questions related to the accuracy or integrity of any part of the work are appropriately investigated and resolved. The research was performed according to the Declaration of Helsinki (as revised in 2013) and the guidelines of the local ethics committee (Guangdong Provincial People's Hospital, NO. GDREC2012143H). All patients have provided written consent.

Open Access Statement: This is an Open Access article distributed in accordance with the Creative Commons Attribution-NonCommercial-NoDerivs 4.0 International License (CC BY-NC-ND 4.0), which permits the noncommercial replication and distribution of the article with the strict proviso that no changes or edits are made and the original work is properly cited (including links to both the formal publication through the relevant DOI and the license). See: https://creativecommons.org/licenses/by-nc-nd/4.0/.

\section{References}

1. Williams B, Mancia G, Spiering W, et al. 2018 ESC/ESH Guidelines for the management of arterial hypertension. Eur Heart J 2018;39:3021-104.

2. Wang Z, Chen Z, Zhang L, et al. Status of Hypertension in China: Results From the China Hypertension Survey: 2012-2015. Circulation 2018;137:2344-56.

3. Whelton PK, Carey RM, Aronow WS, et al. 2017 ACC/ AHA/AAPA/ABC/ACPM/AGS/APhA/ASH/ASPC/ NMA/PCNA Guideline for the Prevention, Detection,
Evaluation, and Management of High Blood Pressure in Adults: A Report of the American College of Cardiology/ American Heart Association Task Force on Clinical Practice Guidelines. Circulation 2018;138:e484-594.

4. January CT, Wann LS, Alpert JS, et al. 2014 AHA/ACC/ HRS guideline for the management of patients with atrial fibrillation: a report of the American College of Cardiology/American Heart Association Task Force on Practice Guidelines and the Heart Rhythm Society. J Am Coll Cardiol 2014;64:e1-76.

5. Marini C, De Santis F, Sacco S, et al. Contribution of atrial fibrillation to incidence and outcome of ischemic stroke: results from a population-based study. Stroke 2005;36:1115-9.

6. Qi Z, Chen H, Wen Z, et al. Relation of Low-Density Lipoprotein Cholesterol to Ischemic Stroke in Patients With Nonvalvular Atrial Fibrillation. Am J Cardiol 2017;119:1224-8.

7. Chugh SS, Havmoeller R, Narayanan K, et al. Worldwide epidemiology of atrial fibrillation: a Global Burden of Disease 2010 Study. Circulation 2014;129:837-47.

8. Conen D, Tedrow UB, Koplan BA, et al. Influence of systolic and diastolic blood pressure on the risk of incident atrial fibrillation in women. Circulation 2009;119:2146-52.

9. Grundvold I, Skretteberg PT, Liestol K, et al. Upper normal blood pressures predict incident atrial fibrillation in healthy middle-aged men: a 35 -year follow-up study. Hypertension 2012;59:198-204.

10. Emdin CA, Wong CX, Hsiao AJ, et al. Atrial fibrillation as risk factor for cardiovascular disease and death in women compared with men: systematic review and meta-analysis of cohort studies. BMJ 2016;532:h7013.

11. Benjamin EJ, Levy D, Vaziri SM, et al. Independent risk factors for atrial fibrillation in a population-based cohort. The Framingham Heart Study. JAMA 1994;271:840-4.

12. Haywood LJ, Ford CE, Crow RS, et al. Atrial fibrillation at baseline and during follow-up in ALLHAT (Antihypertensive and Lipid-Lowering Treatment to Prevent Heart Attack Trial). J Am Coll Cardiol 2009;54:2023-31.

13. Wann LS, Curtis AB, January CT, et al. 2011 ACCF/ AHA/HRS focused update on the management of patients with atrial fibrillation (Updating the 2006 Guideline): a report of the American College of Cardiology Foundation/ American Heart Association Task Force on Practice Guidelines. Heart Rhythm 2011;8:157-76.

14. Dudley SC Jr, Hoch NE, McCann LA, et al. Atrial fibrillation increases production of superoxide by the left 
atrium and left atrial appendage: role of the NADPH and xanthine oxidases. Circulation 2005;112:1266-73.

15. Hu YF, Chen YJ, Lin YJ, et al. Inflammation and the pathogenesis of atrial fibrillation. Nat Rev Cardiol 2015;12:230-43.

16. Meisinger C, Baumert J, Khuseyinova N, et al. Plasma oxidized low-density lipoprotein, a strong predictor for acute coronary heart disease events in apparently healthy, middle-aged men from the general population. Circulation 2005;112:651-7.

17. Steinberg D, Parthasarathy S, Carew TE, et al. Beyond cholesterol. Modifications of low-density lipoprotein that increase its atherogenicity. N Engl J Med 1989;320:915-24.

18. Mourtzinis G, Kahan T, Bengtsson BK, et al. Relation Between Lipid Profile and New-Onset Atrial Fibrillation in Patients With Systemic Hypertension (From the Swedish Primary Care Cardiovascular Database [SPCCD]). Am J Cardiol 2018;122:102-7.

19. Li X, Gao L, Wang Z, et al. Lipid profile and incidence of atrial fibrillation: A prospective cohort study in China. Clin Cardiol 2018;41:314-20.

20. Alonso A, YiN X, Roetker NS, et al. Blood lipids and the incidence of atrial fibrillation: the Multi-Ethnic Study of Atherosclerosis and the Framingham Heart Study. J Am Heart Assoc 2014;3:e001211

21. Watanabe H, Tanabe N, Yagihara N, et al. Association between lipid profile and risk of atrial fibrillation. Circ J 2011;75:2767-74.

22. Haukilahti MA, Holmstrom L, Vahatalo J, et al. Sudden Cardiac Death in Women. Circulation 2019;139:1012-21.

23. Levey AS, Bosch JP, Lewis JB, et al. A more accurate method to estimate glomerular filtration rate from serum creatinine: a new prediction equation. Modification of Diet in Renal Disease Study Group. Ann Intern Med 1999;130:461-70.

24. Dewland TA, Soliman EZ, Yamal JM, et al. Pharmacologic
Prevention of Incident Atrial Fibrillation: Long-Term Results From the ALLHAT (Antihypertensive and LipidLowering Treatment to Prevent Heart Attack Trial). Circ Arrhythm Electrophysiol 2017;10:e005463.

25. Naccarelli GV, Varker H, Lin J, et al. Increasing prevalence of atrial fibrillation and flutter in the United States. Am J Cardiol 2009;104:1534-9.

26. Lopez FL, Agarwal SK, Maclehose RF, et al. Blood lipid levels, lipid-lowering medications, and the incidence of atrial fibrillation: the atherosclerosis risk in communities study. Circ Arrhythm Electrophysiol 2012;5:155-62.

27. Getz GS, Reardon CA. Apoprotein E as a lipid transport and signaling protein in the blood, liver, and artery wall. $\mathrm{J}$ Lipid Res 2009;50:S156-61.

28. White CR, Garber DW, Anantharamaiah GM. Antiinflammatory and cholesterol-reducing properties of apolipoprotein mimetics: a review. J Lipid Res 2014;55;2007-21.

29. Abi-Char J, Maguy A, Coulombe A, et al. Membrane cholesterol modulates $\mathrm{Kv} 1.5$ potassium channel distribution and function in rat cardiomyocytes. J Physiol 2007;582:1205-17.

30. Lundbaek JA, Birn P, Hansen AJ, et al. Regulation of sodium channel function by bilayer elasticity: the importance of hydrophobic coupling. Effects of Micelleforming amphiphiles and cholesterol. J Gen Physiol 2004;123:599-621.

31. Feingold K, Brinton EA, Grunfeld C. The Effect of Endocrine Disorders on Lipids and Lipoproteins. 2000, South Dartmouth (MA): MDText.com, Inc.

32. Cooper DS, Biondi B. Subclinical thyroid disease. Lancet 2012;379:1142-54.

33. Lee HC, Lin YH. The Pathogenic Role of Very Low Density Lipoprotein on Atrial Remodeling in the Metabolic Syndrome. Int J Mol Sci 2020;21:891.
Cite this article as: Huang JY, Liu L, Yu YL, Chen CL, Lo K, Zhang B, Tang ST, Huang YQ, Feng YQ. A nonlinear relationship between low-density-lipoprotein cholesterol levels and atrial fibrillation among patients with hypertension in China. Ann Palliat Med 2020;9(5):2953-2961. doi: 10.21037/ apm-20-451 